\title{
OS INSTRUMENTOS DE LA POLÍTICA EN CIENCIA, TECNOLOGÍA E INNOVACIÓN EN LA ARGENTINA RECIENTE
}

\author{
Instruments of science, technology and \\ innovation policy in recent Argentinian history \\ ID Nerina Fernanda Sarthou*
}

\section{(c) (1)}

* El artículo refleja parte de la investigación desarrollada en el marco del Plan de Trabajo propuesto por la autora como Investigadora Asistente del Consejo Nacional de Investigaciones Científicas y Técnicas (CONICET), Argentina.

** Doctora en Ciencia Política, Investigadora Asistente de CONICET, Docente-investigadora del Centro de Estudios en Problemáticas Internacionales y Locales (CEIPIL), Centro Asociado a la CICPBA, UNICEN, Argentina, Tandil. E-mail: nfsarthou@yahoo.com.ar

Fecha de recepción: 23 de junio de 2017

Fecha de aprobación: 17 de noviembre de 2017

\section{Cómo citar / How to cite}

Sarthou, N. F. (2018). Los instrumentos de la Política en Ciencia, Tecnología e Innovación en la Argentina reciente. trilogía Ciencia Tecnología Sociedad, 10(18), 97-116. 
Resumen: la noción de instrumentos de política ha sido empleada en diversos estudios académicos que analizan el ámbito de la ciencia, la tecnología y la innovación; sin embargo, ello no se ha traducido en un avance en la caracterización de la política. Este artículo posee como propósito analizar la política pública en ciencia, tecnología e innovación en la Argentina reciente, a través de distinguir y caracterizar las tendencias presentes en materia de instrumentos. Para ello, se realiza una caracterización de los mismos de acuerdo con sus objetivos, con los organismos que se encargan de su aplicación, sus destinatarios y con el momento en que fueron creados. El estudio se basa en el procesamiento de información proveniente de la plataforma Políticas CTI gestionada por RICYT. Del análisis se desprende que la mayoría de los instrumentos son implementados por un único organismo del complejo de CTI; que el sector académico es el destinatario principal y que en los últimos quince años la política de CTI en Argentina ha atravesado un proceso de expansión y diversificación de sus instrumentos de significativa relevancia.

Palabras clave: instrumentos; política pública; ciencia, tecnología e innovación.

Abstract: The notion of policy instruments has been used in several academic studies that analyze the field of science, technology and innovation. However, this has not translated into advances in policy characterization. The purpose of this article is to analyze the science, technology and innovation policy in recent Argentinian history by distinguishing and characterizing current trends in instruments. In order to achieve it, the instruments are characterized according to their objectives, the organisms responsible for their application, and the moment of creation. The analysis is based on the information provided by the platform on STI managed by RICYT. The study revealed that most instruments are implemented by a single agency in the STI complex, the academic community is the main target audience and, in the last fifteen years, the STI policy in Argentina has undergone a significant process of expansion and diversification of its instruments.

Keywords: instruments, policy, STI, Argentina. 


\section{INTRODUCCIÓN}

El origen del interés por identificar los instrumentos de las políticas públicas proviene del campo de la ciencia política y de la economía, y se remonta a la década de 1960, cuando los estudiosos advierten el creciente rol que los estados adoptaron en materia de política económica y social tras la Segunda Guerra Mundial. Desde la ciencia política, fue Lowi (1964) quien comenzó identificando distintos tipos de políticas, según el impacto de las mismas. El enfoque de Lowi generó una línea de investigación dentro de la disciplina que significó un recorrido que ha sido abordado en otros trabajos (Sarthou, 2015, 2016). Desde la economía, se realizó uno de los primeros inventarios de instrumentos en la obra de Kirschen y otros (1964), con más de 40 tipos diferentes de instrumentos que prevalecían en la formulación de las políticas económicas. Dicha clasificación se basó en cinco tipos de recursos que implicaban los instrumentos de política: finanzas públicas, dinero y crédito, tipos de cambio, control directo y cambios en el marco institucional.

Luego del proceso global de transformación del estado y del surgimiento del paradigma de la Nueva Gestión Pública, o también referenciado como New Public Management durante la década de 1990, el estudio de los instrumentos de política recibió un nuevo impulso. A partir de la influencia de este marco cognitivo y de una política fiscal restrictiva adoptada por los gobiernos, se introdujeron nuevos formatos en las políticas públicas encaminados a la búsqueda de la «eficiencia» (Souza, 2006).

Desde el campo de los estudios sociales de la ciencia y la tecnología, un número relevante de académicos (Albornoz y Gordon, 2010; Hurtado, 2010; Porta y Lugones, 2011; Mallo, 2011; Aristimuño y Aguiar,
2015) ha llegado a la conclusión de que durante la década de 1990 se produjo una reconfiguración institucional en el sector, que afectó hasta la actualidad la orientación de las políticas públicas en ciencia y tecnología. En particular, se destacaron como hitos la sanción de la Ley de Promoción y Fomento de la Innovación en 1992; la creación del Fondo Tecnológico Argentino (FONTAR en 1994, el Fondo para la Investigación Científica y Tecnológica (FONCYT) y la Agencia Nacional de Promoción Científica y Tecnológica (ANPCYT) en 1996; la elaboración de planes nacionales de mediano plazo como práctica institucionalizada y la consolidación de una relación fuerte con el Banco Interamericano de Desarrollo (BID) ${ }^{1}$, como organismo financiador y principal interlocutor internacional en la agenda de las políticas e instrumentos de promoción de la CyT en la Argentina (Aguiar, Aristimuñoy Magrini, 2015). A pesar de estos aportes, no se ha profundizado en las continuidades o los cambios en la política a partir de transformaciones que se han producido a nivel de los instrumentos de política pública, mediante los cuales las instituciones fomentan la ciencia, tecnología e innovación (CTI) en el país.

El estudio de los instrumentos de política en CTI en Argentina comenzó hacia la década en 1970 en el marco del desarrollo del Proyecto de Instrumentos de Política Científica y Tecnológica coordinado por Francisco Sagasti. Los primeros aportes para el caso argentino fueron generados por Eduardo Amadeo (Sagasti, 2015²), no obstante, no se advierte una línea de reflexión que haya logrado continuidad, es decir, los estudios sobre los instrumentos en CTI presentan una dinámica que no ha conducido al surgimiento y empleo de un enfoque propio.

El presente trabajo tiene como objetivo analizar los instrumentos de la política pública en CTI

${ }^{1}$ Para mayor detalle sobre la influencia del BID en la política en CTI en la Argentina ver: Aristimuño, Federico, Aguiar, Diego y Magrini, Nicolás. (2017). «Transferencia de modelos institucionales o redes de asuntos de expertos? Análisis de un préstamo para ciencia y tecnología en Argentina del Banco Interamericano de Desarrollo durante los noventa». Estudios Sociales del Estado, 3(5), 99-131. Aguiar, Diego, Aristimuño, Francisco y Magrini, Nicolás. (2015). «El rol del Banco Interamericano de Desarrollo (BID) en la re-configuración de las instituciones y políticas de fomento a la ciencia, la tecnología y la innovación de la Argentina (1993-1999)». Revista Iberoamericana de Ciencia, Tecnologia y Sociedad. Lugones, Guillermo, Porta, Federico y Codner, Darío (2014) Insights into the Impact of BID’s Technology Modernization Program on Argentina's STI Policy. En G. Crespi y G. Dutrenit eds. Science, Technology and Innovation Policies for Development, Springer. pp. 63 - 86. Abeledo, Carlos (2000). Análisis del financiamiento del Banco Interamericano de Desarrollo para ciencia y tecnología. 
LOS INSTRUMENTOS DE LA POLÍ́TICA EN CIENCIA,

TECNOLOGÍA E INNOVACIÓN EN LA ARGENTINA RECIENTEA

100 en la Argentina reciente. Se busca distinguir y caracterizar las tendencias presentes en materia de instrumentos y lograr una aproximación al tipo de política que se ha implementado en el país. El artículo se estructura en cuatro secciones. En la primera sección se realiza un recorrido por la literatura dentro del campo de los estudios de la política científica que han empleado y reelaborado la noción de instrumentos de política en CTI. En la segunda sección, se realiza una presentación de la metodología empleada y una caracterización general de los instrumentos de CTI implementados en el país. En la tercera sección, se analizan los instrumentos de la política CTI con base en los organismos que se ocupan de su implementación, de acuerdo con quiénes son sus destinatarios y con base en el momento en que fueron creados. Finalmente, se presenta una breve síntesis de los resultados de la investigación.

\section{EL EMPLEO DE LA NOCIÓN DE INSTRUMENTOS DE POLÍTICA EN EL ESTUDIO DE LA POLIITICA EN CIENCIA, TECNOLOGÍA E INNOVACIÓN}

Desde el campo de estudios de política científica y tecnológica en América Latina, la noción de «instrumento de política» fue empleada por primera vez por Francisco Sagasti, como coordinador del Proyecto de Instrumentos de Política Científica y Tecnológica financiado por el Centro Internacional de Investigaciones para el Desarrollo (IDRC), organismo de origen canadiense. Dicho proyecto constituyó un esfuerzo de cooperación internacional que durante la década de 1970 emprendieron diez países en desarrollo, entre los que se encontraban Argentina, México, Perú, Colombia, Venezuela y Brasil. En el transcurso del desarrollo del proyecto, surgió un documento específico que Sagasti preparó junto a
Aráoz (1976) y a un grupo de expertos -mediante una línea de financiamiento del Departamento de Asuntos Científicos de la Organización de Estados Americanos (OEA)- referido a una serie de «pautas metodológicas» para ser utilizadas en el análisis de cada contexto nacional y en el proceso de toma de decisiones en la materia.

En este documento, los instrumentos de política fueron definidos como «el conjunto de modos y medios utilizados para poner en práctica una política determinada (...) el vehículo mediante el cual los que tienen a su cargo la formulación y ejecución de las políticas ejercen su capacidad de influir en las decisiones que toman los demás» (Sagasti y Aráoz, 1979, p. 12). De acuerdo con estos autores, un instrumento comprende un dispositivo legal, una estructura de organización y un conjunto de instrumentos operacionales. Asimismo, afirman que existen actores asociados a la toma de decisiones, el diseño y el uso de los instrumentos de política que son un factor clave en el análisis.

Respecto a su clasificación, para Sagasti y Aráoz (1979), los instrumentos pueden catalogarse según busquen afectar la demanda, la oferta o los vínculos entre ambas. A su vez pueden ser directos o indirectos dependiendo de si se refiere explícitamente a funciones y actividades científicas y tecnológicas o no; discriminados o indiscriminados según el carácter de su aplicación; positivos o negativos, basados en si tienen como meta estimular, facilitar o inducir determinadas acciones o si restringen, prohíben o desalientan ciertas actividades; y recuperando las nociones de Herrera (1972), los instrumentos pueden ser explícitos o implícitos.

Bastantes años después, Albornoz (2007) elaboró un informe en el marco de la Red Iberoamericana

\footnotetext{
${ }^{2}$ El economista Eduardo Amadeo fue el coordinador del equipo argentino formado por dos investigadores y varios consultores que trabajaron temas específicos. La ubicación inicial del equipo fue el Departamento de Economía de la Universidad Católica Argentina; después de unos meses se trasladó a la sucursal Argentina del Consejo Latinoamericano de Ciencias Sociales (CLACSO). El equipo argentino se enfocó en dos ramas de la industria: la referida a la maquinaria industrial y la industria petroquímica, aunque también estudió otros problemas. Por ejemplo, los informes incluyen un documento sobre el contenido tecnológico del plan de desarrollo trienal (1974-77), un estudio de la estructura industrial argentina, una descripción y análisis de instrumentos de política tecnológica en Argentina, un estudio del sistema regulatorio de importaciones de tecnología y un informe sobre la asistencia técnica internacional como instrumento de la política de tecnología (Sagasti, 2015).
} 
de Indicadores de Ciencia y Tecnología (RICYT) sobre las políticas vigentes en la actualidad en América Latina, en el cual identificó cinco grupos de instrumentos en función de sus objetivos: 1) para la generación de conocimiento científico básico y aplicado; 2) para la generación de productos y servicios de alto valor agregado; 3) para la formación de recursos humanos en ciencia, tecnología e innovación; 4) para el desarrollo de áreas tecnológicas estratégicas para el país; y 5) para la generación de redes de articulación del sistema.

Esta noción es retomada por Emiliozzi, Lemarchard y Gordon (2009) en el marco de un proyecto de construcción de un inventario de instrumentos de política financiado por el BID. Dentro del marco de un convenio de cooperación técnica entre el BID y la RICYT, se introduce nuevamente la necesidad de recolectar y sistematizar la información referente a los instrumentos de PCTI, que son implementados por los distintos países de América Latina y el Caribe. A partir de la definición de Sagasti y Araóz (1976) Emiliozzi, Lemarchard y Gordon (2009), identificaron diversos tipos de instrumentos operacionales, entendidos como aquellos que explícitamente transforman las políticas en metas concretas.

Producto de la colaboración entre el BID y la RICYT, y con el propósito de reunir en un mismo sistema de forma normalizada la información acerca de las políticas y los instrumentos en CTI, la RICYT lanzó la Plataforma de políticas e instrumentos en ciencia tecnología e innovación -www.politicasCTI.net-. La plataforma permite la consulta de información cualitativa sobre los sistemas institucionales, las políticas y los instrumentos en ciencia, tecnología e innovación implementados por los países de América Latina y el Caribe.

La principal herramienta de la plataforma consiste en una base de datos dinámica que sistematiza los instrumentos de políticas en CTI implementados desde 2008 por los países de la región. Los instrumentos fueron clasificados en tres niveles de análisis, y según cada país, a partir de las siguientes dimensiones: a) ejes (objetivo o finalidad general); $b$ ) categorías (subdivisión de cada eje) y c) descripción analítica. De esta manera, la base de datos dinámica permite realizar tres tipos de consulta: por país, por eje y por categoría para todos los países y, consultas más específicas sobre qué países están utilizando determinado tipo de instrumento (categoría) dentro de cada eje de políticas. En una segunda fase del proyecto, la base fue actualizada con el apoyo del Observatorio CTS de la Organización de Estados Iberoamericanos (OEI).

También, utilizando como fuente de datos la plataforma de políticas CTI, desde el centro argentino CIECTI se elaboró un informe sobre «Las políticas de CTI en países emergentes» (2015) que explora, entre otras cuestiones, los instrumentos de política pública. Lo que aporta este informe, además de un breve análisis de los instrumentos de algunos países de América Latina, es una indagación más profunda de un conjunto de instrumentos aplicados en otros contextos pero que pueden ser considerados para su implementación en Argentina. Se describe y evalúa la aplicabilidad de siete instrumentos de política CTI: la innovación autóctona (Innofund en China); la innovación frugal (Fondo Indio para la Innovación Inclusiva, en India); los fondos de capital de riesgo (Banco de Desarrollo de las pyme, en India, Programa Innovar en Brasil y Yozma, en Israel); los fondos sectoriales (diversos fondos en Brasil); las fundaciones de estímulo a la investigación (Fundación del Estado de San Pablo en Brasil); los conglomerado de empresas públicas de I+D (Rostec, en Rusia) y los instrumentos para la formación de recursos humanos (Ciencia sin frontera, en Brasil).

Por su parte, también Baptista (2015) presentó un análisis de las tendencias recientes en materia de instrumentos de política CTI en América Latina y el Caribe, empleando la plataforma mencionada. Puntualmente, en su trabajo realiza una evaluación de los niveles de complementariedad y de diversidad de los 
LOS INSTRUMENTOS DE LA POLÍ́TICA EN CIENCIA,

TECNOLOGÍA E INNOVACIÓN EN LA ARGENTINA RECIENTEA

102 instrumentos implementados en la región a partir del empleo de la Plataforma politicaCTI. net y retoma el documento elaborado por Emiliozzi, Lemarchard y Gordon (2009). Entre sus conclusiones, esta autora señala que hasta 2014, la mayor parte los instrumentos que se implementaron tenían como objetivo el fomento de la innovación y el apoyo a la formación de recursos humanos; luego le seguían con cantidades muy similares entre sí, los instrumentos de fomento a la investigación y al desarrollo de áreas estratégicas y, los orientados a la articulación del sistema. A nivel comparativo, Brasil concentraba más del $25 \%$ de los instrumentos implementados en toda la región, siguiendo en orden descendente Argentina, México, Chile y Uruguay. Estos cinco países en conjunto concentraban más del $65 \%$ de los incentivos públicos a actividades de CTI que se implementan en toda América Latina. Lo anterior da cuenta que la tendencia general es que los países más grandes de la región, tanto en términos poblacionales como de producto sean los que despliegan un mayor número de instrumentos de promoción de las actividades de CTI. En este mismo sentido, Baptista (2015) destaca que se verifica una alta correlación positiva entre la cantidad de instrumentos y la inversión en $\mathrm{I}+\mathrm{D}$ en relación al PBI de los diferentes países.

Por otra parte, a fines del año 2010, la Oficina Regional de Ciencia para América Latina y el Caribe de la UNESCO con sede en Montevideo, publicó el documento «Sistemas nacionales de ciencia, tecnología e innovación en América Latina y el Caribe» (Lemarchard, 2010), un estudio evolutivo de las políticas de CTI en América Latina y el Caribe, que ofrece el análisis de las últimas seis décadas, junto con un inventario de los sistemas nacionales de ciencia, tecnología e innovación de los países de la región. Conjuntamente, la Oficina lanzó SPIN³ una plataforma de información sobre política científica que cuenta -en la actualidad- con un inventario con la descripción detallada con más de 1000 instrumentos de política científica y tecnológica aplicados por los estados de la región, clasificados de acuerdo a 15 objetivos y metas estratégicas, 15 tipos de mecanismos y 23 categorías de beneficiarios. Asimismo, cada instrumento se describe mediante una serie de datos específicos.

Empleando la base de datos SPIN, Lemarchard (2015) elaboró recientemente un diagnóstico sobre la situación de la ciencia, la tecnología y la innovación en la región de América Latina y el Caribe, que fue incorporado en el informe mundial de la UNESCO sobre la ciencia hacia 2030. Entre sus conclusiones, este autor señala que los instrumentos más tradicionales para promover la investigación científica en la región son los subsidios competitivos y los centros de excelencia. No obstante, en las últimas dos décadas, la mayoría de los países latinoamericanos han creado fondos específicos para la investigación y la innovación competitivas. Estos, denominados en general fondos sectoriales son un ejemplo de la diversidad de instrumentos de política sofisticados que promueven la investigación y la innovación en América Latina, incluso si estos instrumentos han demostrado ser más eficaces en algunos países que otros.

Por último, se destaca la labor realizada desde la Comisión Económica para América Latina (CEPAL), en conjunto con la Agencia Alemana de Cooperación GTZ ${ }^{4}$. En el marco de la División

${ }^{3}$ La idea y estructura de la plataforma latinoamericana dio lugar a la conformación a nivel mundial del Observatorio Global de Instrumentos de Política en Ciencia, Tecnología e Innovación denominado Go Spin de la UNESCO. Aunque en la actualidad la plataforma no está disponible de manera abierta y en línea, desde su presentación en 2012 se publicaron seis volúmenes sobre diferentes países dentro de la colección de «Perfiles Nacionales» preparados por el observatorio: Bostwana, Zimbadwe, Malawi, Ruanda, Israel y Guatemala. 
de Desarrollo Productivo y Empresarial de la CEPAL, Annalisa Primi y Mario Cimoli coordinaron el desarrollo de un portal web de políticas CTI denominado CyT-DES que contiene una base de datos (http://www.cepal.org/iyd/) con un inventario de instrumentos de política en el área. El sitio está organizado en una sección sobre «Ideas, experiencias y desafíos para la formulación de políticas de CyT» y en una sección de «Asistencia técnica» dedicada a explorar ámbitos específicos de la formulación, implementación y evaluación de políticas. Dentro de la sección Ideas, se puede encontrar el apartado «INFO-INSTRUMENTOS», una base de datos de instrumentos de política de CTI que contiene información sobre ejemplos de instrumentos de política para un universo de 45 países de distintas regiones. La base de datos no pretende mostrar todos los instrumentos presentes en cada país sino destacar las diferentes formas de apoyar el desarrollo científico y tecnológico.

Hasta aquí, puede decirse que la noción de instrumento de política dentro de los estudios de política científica ha tenido como propósito central sistematizar la información recolectada en los distintos países, proveer de datos completos para la realización de estudios de impacto intertemporales a nivel país y generar bases de datos de sostenimiento y actualización accesible; también debe destacarse el rol clave que han tenido los organismos internacionales en el fomento de este tipo de estudios sobre la región (Sarthou, 2015).

\section{LOS INSTRUMENTOS DE LA POLITICA DE CIENCIA, TECNOLOGÍA E INNOVACIÓN EN ARGENTINA}

\section{METODOLOGÍA}

Para la investigación se consideraron los siete ejes referidos a los objetivos generales que persigue cada instrumento y las 16 categorías de instrumentos de política de $\mathrm{CTI}^{5}$ propuestos por RICYT en la plataforma Políticas CTI (Tabla 1). Cabe destacar que esta clasificación fue elaborada a fines del año 2016, producto de una revisión llevada a cabo por el equipo de trabajo que gestiona la plataforma. En dicha oportunidad, se advirtió que muchos de los instrumentos que se habían relevado en los países de la región no estaban coincidiendo del todo con las categorías preexistentes, que habían sido redefinidas por Emiliozzi, Gordon y Lemarchand en 2009. Es así que luego de un proceso de análisis y debate, acompañados por el Coordinador de la RICYT, Rodolfo Barrere, se decidió avanzar en la redacción de un nuevo documento metodológico que propone ejes y categorías más específicos de acuerdo con las características de los instrumentos de políticas de CTI.

\footnotetext{
${ }^{4}$ GTZ (Cooperación Técnica Alemana) fue fundada en 1975 por el Gobierno Federal Alemán, como una empresa de propiedad estatal dedicada a la cooperación internacional al desarrollo. En 2011 se creó la Agencia Alemana para la Cooperación Internacional para el Desarrollo (GIZ) mediante la fusión de tres organizaciones históricas de la cooperación alemana: la GTZ, la Agencia Alemana de Desarrollo Internacional (DED) y la Sociedad para la Formación y el Desarrollo. La GIZ está constituida jurídicamente como empresa estatal y actúa principalmente como órgano de implementación a nivel técnico, es decir, cooperación técnica en nombre del Ministerio de Cooperación Económica y Desarrollo (BMZ). Ver para más detalles Kerstin Maier, 2012. El modelo alemán de Cooperación Internacional para el Desarrollo sobre la cooperación internacional alemana. Documento de Trabajo, COIBA, Universidad de Cantabria. Disponible en: http://www.ciberoamericana.com/pdf/Briefing9.pdf

${ }^{5}$ Cabe resaltar que la plataforma empleada como fuente de datos contiene el esquema de promoción CTI en el país proveniente del nivel Gobierno nacional, es decir, que contempla instrumentos cuya autoridad de aplicación se encuentra en el ámbito del Estado Nacional, ello requiere aclarar que el presente estudio no incluye en el análisis los instrumentos de los niveles de Gobierno provincial y municipal. Asimismo, la plataforma no contempla instrumentos propios de algunos organismos argentinos de relevancia, como el Instituto Nacional de Tecnología Industrial (INTI), el Instituto Nacional de Tecnología Agropecuaria (INTA) y las universidades.
} 
LOS INSTRUMENTOS DE LA POLÍ́TICA EN CIENCIA,

TECNOLOGÍA E INNOVACIÓN EN LA ARGENTINA RECIENTEA

Investigación + Desarrollo

Fondos de promoción a la investigación científica y tecnológica

Incentivos docentes a la investigación científica y tecnológica

\section{Infraestructura}

Fondos para creación y mejora de equipamiento e infraestructura

Fondos para la creación de cluster, polos tecnológicos e incubadoras de empresas

Innovación

Fondos de promoción a la innovación y la competitividad de las empresas

Programas para la creación y fomento de PYME

Programas de apoyo a la incorporación de investigadores y becarios en empresas

Recursos Humanos

Becas para estudios de grado, posgrado y postdoctorado

Programas de creación y apoyo a posgrados

Programas de capacitación técnica

Áreas Estratégicas

Programas de áreas prioritarias

Fondos sectoriales

Vinculación

Estructuras de interfase

Vinculación internacional de investigadores y becarios nacionales

Visita de investigadores extranjeros al país

Cultura Científica

Programas de cultura científica

Fuente: Plataforma Web, www.politicascti.net.

\section{RESULTADOS Y DISCUSION}

A partir del «Reporte de Instrumentos», que genera la plataforma, los instrumentos de política que implementó el Estado argentino hasta el año 2016 sumaron un total de $97^{6}$, superando ampliamente el promedio regional de 27 instrumentos por país en 2014 y ubicándose como el segundo país con mayor número, por debajo de Brasil (Baptista, 2015). La mayor parte de dichos instrumentos (Figura 1) tenían como gran objetivo el fomento de la innovación (25\%) y el apoyo a la formación de recursos humanos $(23 \%)$.
En un segundo nivel, en cuanto a cuantía, se ubicaban los instrumentos de desarrollo de áreas estratégicas (19\%) y de mejoramiento de la infraestructura $(16 \%)$. En un tercer nivel, se encontraban los instrumentos para el fomento de la I+D (11\%) y, por último, aquellos para estimular la vinculación y el desarrollo de la cultura científica, con cantidades similares (3\%).

En cuanto a los tipos de instrumentos utilizados, dentro de las 16 categorías, Argentina implementa mayormente cinco tipos de instrumentos (Figura 2): Fondos de promoción de la innovación y la

\footnotetext{
${ }^{6}$ En la plataforma politicascti.net se contabilizaron 105 instrumentos para el caso argentino, no obstante, se han eliminado ocho instrumentos por encontrarse reiterados. Estos son: 1) Proyectos de adecuación y/o mejora de infraestructura, 2) Consejerías tecnológicas individuales y 3) Consejerías tecnológicas grupales (ambas pasaron a denominarse Asistencias Tecnológicas en 2016, de allí la reiteración), 4) Programa de fomento de la inversión emprendedora en tecnología, 5) Aporte No Reembolsable Bio, Nano, Tics, 6) Ideas proyectos Concertados con Empresas (IP-CE 2016-2019), 7) Aporte No Reembolsable Tecnología. En la categoría Recursos Humanos se suprimió el instrumento 8) SENESCYT - Becas cooperación internacional, por pertenecer al Ecuador.
} 
competitividad de las empresas (19), Becas para estudios de grado, posgrado y posdoctorado (15), Fondos para creación y mejora de equipamiento e infraestructura (13), Fondos Sectoriales (13) y
Fondos de promoción a la investigación científica y tecnológica (10). Solo estas cinco categorías suman un total de 73 instrumentos, representando el $72 \%$ del total de los mismos.

Figura 1. Distribución de instrumentos de política CTI en Argentina Según Eje

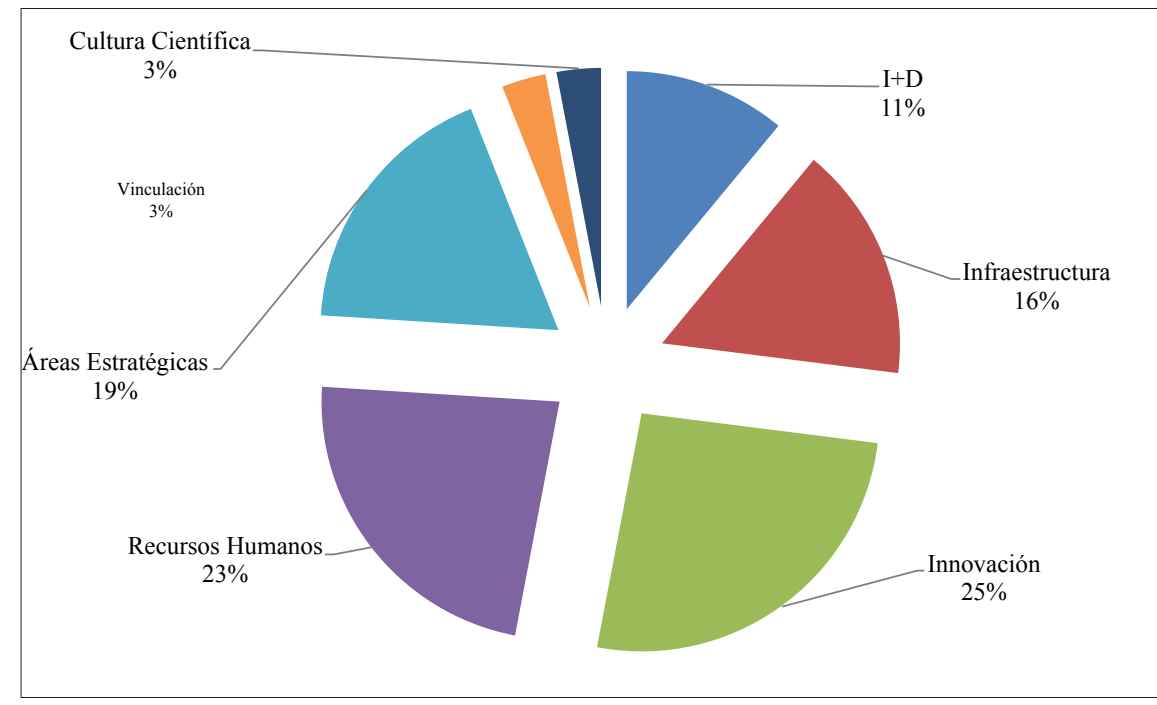

Fuente: elaboración propia.

Figura 2. Distribución de insrumentos de políticas CTI en Argentina según categoría

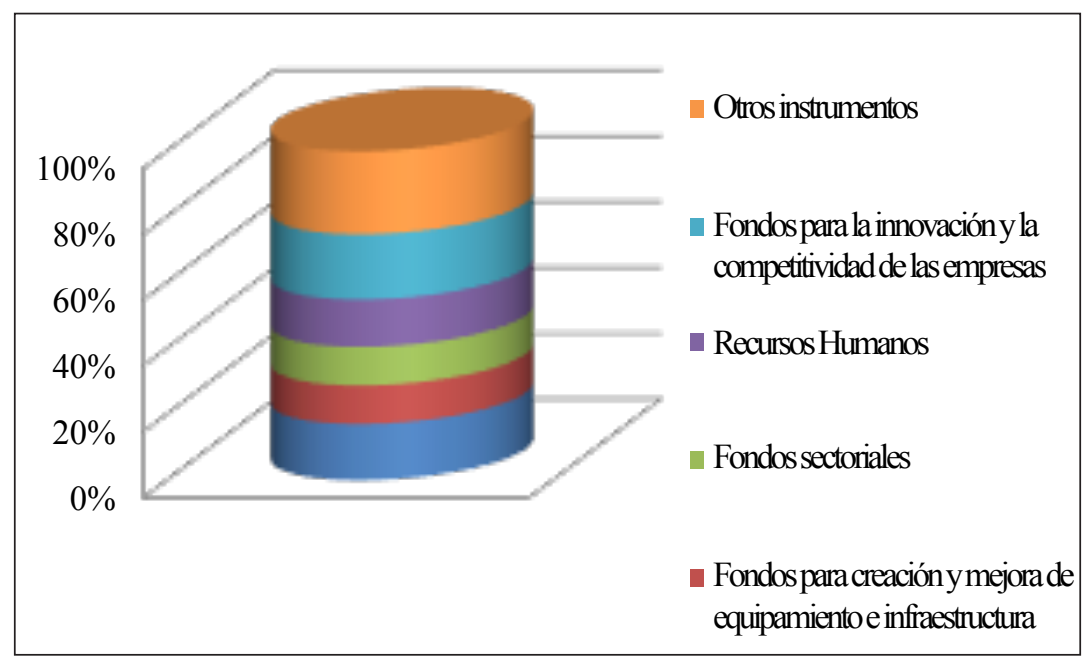

Fuente: elaboración propia. 
LOS INSTRUMENTOS DE LA POLÍTICA EN CIENCIA,

TECNOLOGÍA E INNOVACIÓN EN LA ARGENTINA RECIENTEA

106 Caracterización de los instrumentos de políitica CTI

A continuación, se presenta una aproximación a la oferta de instrumentos relevada en la plataforma www.politicascti.net, la cual es complementada a través de una profundización del análisis a partir de dimensiones como los organismos encargados de la aplicación de los instrumentos, los destinatarios finales y la edad de los mismos o su momento de aplicación.

a) Los instrumentos de política CTI según los organismos de implementación

Si se examinan los organismos encargados de implementar cada instrumento de política (Figura 3) se obtiene que la mayor parte de ellos (62 \%) son administrados por la ANPCyT, seguidos por el MINCYT (25\%) y el CONICET (12\%). Existe un único instrumento-relevado por la plataforma-que es implementado por el Ministerio de Educación y Deportes (MEyD), a través de la Secretaría de Políticas Universitarias (SPU), que es el Programa de Incentivos a Docentes-Investigadores de Universidades, implementado desde 1994 en todas las instituciones de educación superior de gestión pública y tiene como objetivo central el fomento de la investigación científica como parte de la carrera académica (Sarthou, 2015).

La cantidad y la diversidad de instrumentos implementados dan cuenta del desarrollo de importantes capacidades institucionales de cada organismo de ejecución. Para quela implementación sea exitosa, el marco institucional tiene que ser predecible con el fin de permitir la experimentación, el monitoreo y la evaluación de las políticas y, se debe contar con una masa crítica de capital humano tanto en la agencia responsable como en su sistema de apoyo (por ejemplo, evaluadores, revisores pares y no pares, etcétera).

Figura 3. Instrumentos de política CTI según organismo de implementación

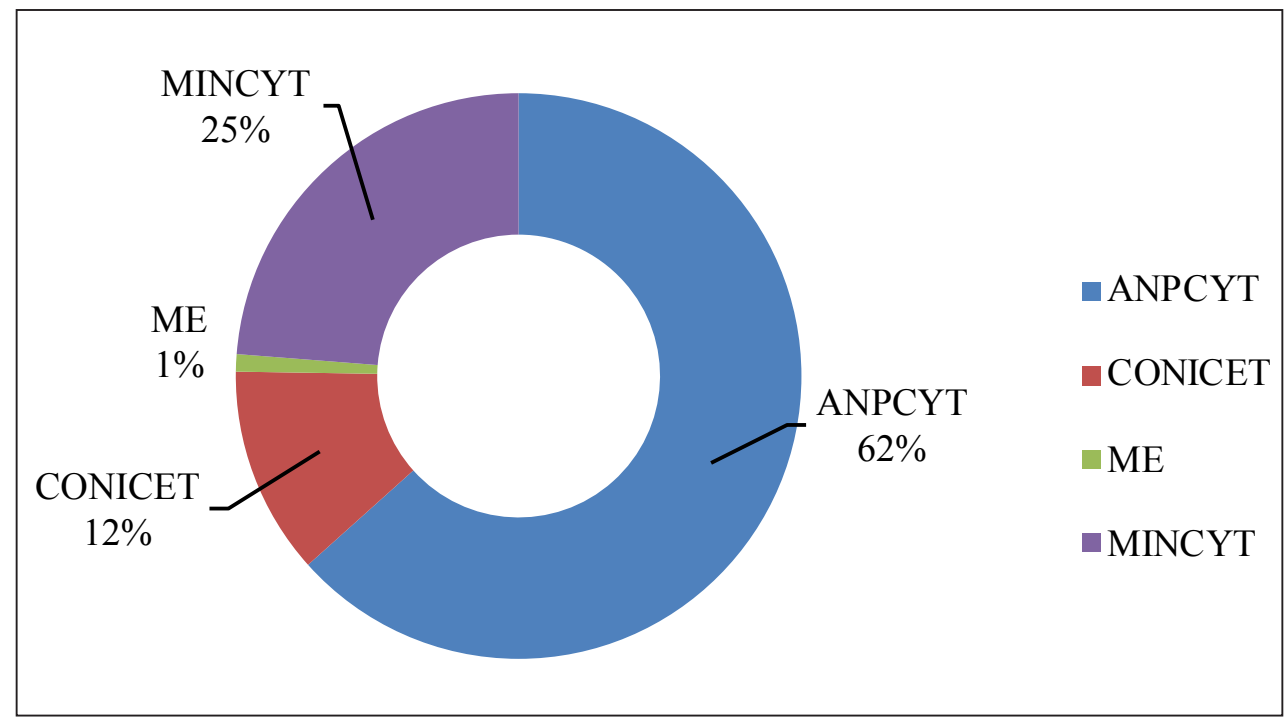

Fuente: elaboración propia.

Los cuatro organismos presentan una trayectoria muy diferente en relación a la política en CTI argentina. La ANPCyT es el organismo que reúne un mayor número de instrumentos. Fue creada en 1996 como un organismo desconcentrado de la Secretaría en Ciencia y Tecnología, y luego del MINCyT, lo que supone que posee cierta autonomía para llevar adelante su misión, aunque depende jerárquicamente del ministerio: no puede elegir a sus propias autoridades, ni poseer 
patrimonio propio (Emiliozzi, 2012). La misión principal de la ANPCYT es la de organizar e implementar instrumentos para la promoción y el fomento del desarrollo científico y tecnológico y de la innovación tecnológica en el país. Sirve a dos clases de clientes, a los investigadores y grupos de investigadores de universidades, institutos y centros de investigación públicos y privados, y a los emprendedores y a las empresas que quieren modernizarse tecnológicamente o hacer nuevos desarrollos tecnológicos. No obstante, esta diferenciación, la ANPCYT también ha impulsado líneas de financiamiento para facilitar la interacción entre la academia y el sector productivo. Cuenta con una importante disponibilidad de recursos, en parte surgidos de acuerdos internacionales de financiamiento y canalizados a partir de cuatro fondos o «instrumentos-marco» (Sarthou, 2015): el FONCYT, el FONTAR, el Fondo Fiduciario de Promoción de la Industria delSoftware (FONSOFT) ${ }^{7}$ y el Fondo Argentino Sectorial (FONARSEC) ${ }^{8}$.

Los instrumentos que implementa la ANCPyT poseen una alta heterogeneidad entre ellos, porque están dirigidos a diferentes destinatarios y tienen muy distintos objetivos; asimismo, son los más complejos de implementar debido a que atraviesan un sistema de evaluación con distintas etapas, que involucra una amplia variedad de tipos de evaluadores.

De otro lado, se encuentra el MINCYT, creado en diciembre de 2007 a pocos días de la asunción de la elegida para sumir el cargo como Presidente, Cristina Fernández de Kirchner, separándolo del Ministerio de Educación, del que continúa dependiendo la política universitaria. Los instrumentos que gestiona este organismo, si bien también son muy diferentes entre sí, comparten un rasgo distintivo: la mayor parte de ellos están destinados al sector académico, ya sea para la creación y mejora de equipamiento e infraestructura, para la capacitación técnica, para formación de recursos humanos o para fomentar la vinculación internacional. El único instrumento destinado al sector empresarial es el Programa de Fomento de la Inversión Emprendedora en Tecnología (PROFIET), complementado por un instrumento que busca fomentar el desarrollo emprendedor: la «Semana nacional del emprendedor tecnológico», de carácter anual.

Entre los instrumentos de este organismo, se destacan aquellos para promover la cultura científica. El MINCYT es el único de los organismos relevados por la plataforma, que cuenta con este tipo de instrumentos. En particular, implementa diversas iniciativas de divulgación, proyectos de cultura científica y, el programa nacional de popularización de la ciencia y la innovación, que se encarga de gestionar una multiplicidad de actividades.

Por último, se encuentran aquellos instrumentosque implementa el Consejo Nacional de Investigaciones Científicas y Técnicas (CONICET) ${ }^{9}$. Creado en 1958, se caracteriza por ser el principal organismo para la formación de investigadores en la Argentina (Emiliozzi, 2012). Desde sus orígenes cuenta con un programa de becas internas de distintas categorías que a lo largo del tiempo se fueron ampliando. Una novedad fue en 1987 la creación de la beca de doctorado, un instrumento que representa un financiamiento importante en términos de

\footnotetext{
${ }^{7}$ El FONSOFT fue creado en 2004 mediante la Ley de Promoción de la Industria del Software y promueve el fortalecimiento de las actividades de producción de software nacional mediante créditos y subsidios, alineado con los objetivos definidos en el Plan Estratégico «Argentina Innovadora 2020», la demanda del mercado nacional y las tendencias internacionales en materia de innovación tecnológica.

${ }^{8}$ El FONARSEC fue creado en 2009 con el propósito de desarrollar capacidades críticas en áreas de alto impacto potencial y transferencia permanente al sector productivo, para mejorar la competitividad y la solución a problemas que se originan en demandas de la sociedad, las empresas y el Estado. ${ }^{9}$ Históricamente el CONICET implementó cuatro instrumentos principales: las becas para la formación de investigadores, la carrera del investigador científico-tecnológico y del personal de apoyo, los subsidios para proyectos y la creación de institutos (Calderari et. al, 1990). La base de datos de la plataforma policticascti, solo contiene información sobre el instrumento de becas para la formación de investigadores, es decir, en el caso de CONICET quedan por fuera tres tipos de instrumentos que el organismo viene implementando hace varias décadas.
} 
LOS INSTRUMENTOS DE LA POLÍTICA EN CIENCIA,

TECNOLOGÍA E INNOVACIÓN EN LA ARGENTINA RECIENTEA

108 cantidad de beneficiarios y montos adjudicados. $\mathrm{Al}$ año siguiente el sistema quedaba organizado en dos niveles: becas de nivel cuaternario, las cuales incluían las becas de pre-iniciación, iniciación, perfeccionamiento, doctorado y formación superior; y becas posdoctorales o de formación equivalente, que incluían las becas posdoctorales, las industriales y las de investigador formado (Calderari et. al, 1990). En la actualidad, el sistema contiene una diversidad mayor de becas internas y externas.

Los instrumentos del CONICET son lo más antiguos relevados y se distinguen por ser destinados al sector académico; específicamente son becas para la formación de recursos humanos. Existe un único tipo de beca que involucra la participación del sector empresarial ya que es cofinanciada entre CONICET y una empresa.

Respecto a este tipo de instrumentos, el segundo organismo de importancia a nivel nacional lo constituye el FONCYT -perteneciente a la ANPCYT-. Dicho organismo ofrece becas de dedicación exclusiva de nivel inicial y superior, en el marco de los proyectos de las diferentes líneas de financiamiento. También otros fondos de la ANCPYT -FONSOFT y FONARSEC- poseen el instrumento de becas para la formación de recursos humanos. b) Los instrumentos y los destinatarios

En el ámbito de la política CTI, en principio, los instrumentos se caracterizan por distribuir recursos financieros, es decir, son instrumentos de incentivos dirigidos a eliminar o reducir la restricción de financiamiento. Si bien, entonces, todos constituyen una transferencia de recursos, revisten distintas características según sus destinatarios.

Un dato poco explorado sobre los instrumentos de política CTI en Argentina resulta ser a quienes van dirigidos. En este trabajo se han identificado tres sectores destinatarios: a) sector académico, incluye a investigadores individuales, universidades, institutos de investigación, instituciones privadas sin fines de lucro; b) sector empresarial, incorpora a empresas de diversos tamaños y provenientes tanto del sector público como del sector privado y; c) sector académico y empresarial, existe un porcentaje de instrumentos que están dirigidos a ambos sectores e, incluso, en algunos casos la participación en conjunto es requisito ineludible (Figura 4). En el caso de la política de CTI en Argentina, la mayor parte de los instrumentos se encuentran dirigidos a afectar al sector académico (55\%), no obstante, un número importante de ellos tiene como destinatario a actores del sector empresarial (29\%) y otro, a ambos sectores (16\%). 


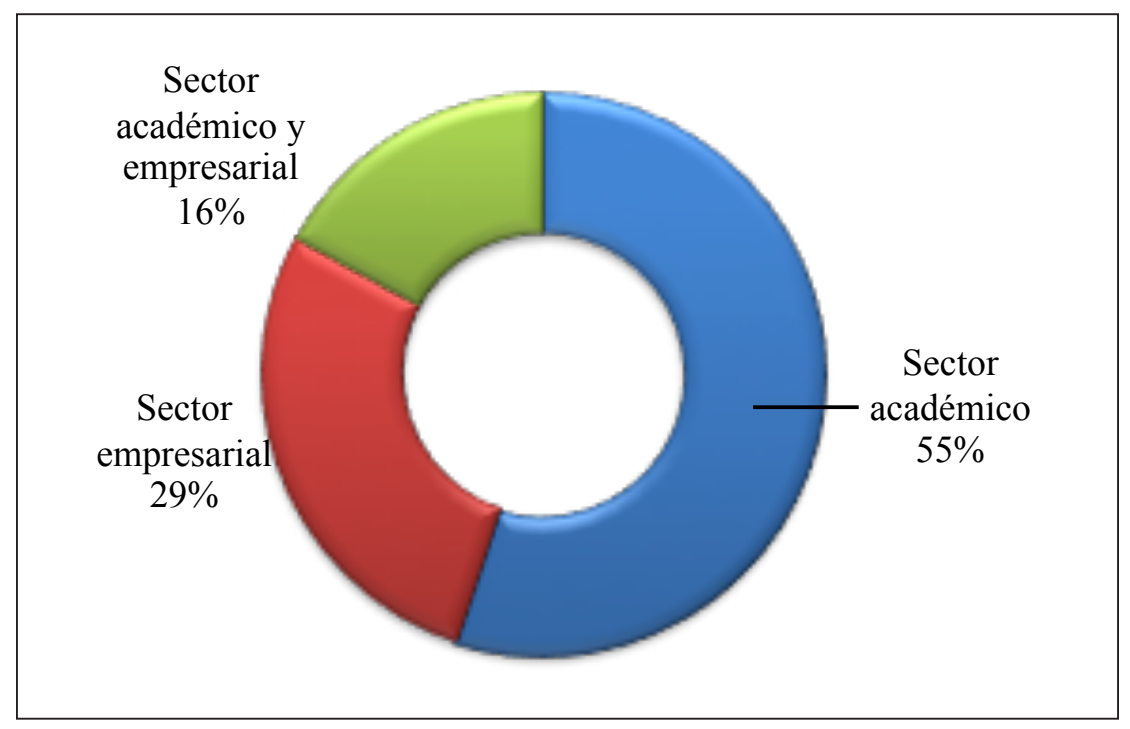

Fuente: elaboración propia.

Aquellos instrumentos que van dirigidos al sector empresarial se caracterizan por ser financiamiento para proyectos que revisten la forma de Aportes No Reembolsables (ANR), créditos con tasas bajas y

créditos fiscales; los tres son recursos financieros, pero difieren en la modalidad de su entrega.

Los ANR están constituidos por financiamiento, que quien recibe no tiene de devolver; dependiendo de la fuente de financiamiento, cada ARN conlleva sus propios términos y condiciones. Estos son instrumentos basados en la premisa de que el Estado comparte el riesgo con el sector privado, financiando gran parte del proyecto. Las empresas objeto de estos programas son por lo general pequeñas o nuevas según criterios establecidos respecto del tamaño de sus ventas y empleados. En términos generales, estos instrumentos buscan financiar proyectos dirigidos al mejoramiento de la productividad del sector privado a través de la innovación tecnológica. No obstante, los objetivos específicos varían según el instrumento, pudiéndose mencionar los siguientes propósitos para el caso de la política CTI en Argentina:

- El fomento de la Investigación y Desarrollo (I+D), por ejemplo, financiando la creación o fortalecimiento de unidades de I+D en empresas a través de la incorporación de investigadores y equipamiento de investigación o la creación o fortalecimiento de plataformas tecnológicas.

- La promoción de la asociatividad entre PYME proveedoras o clientes de una gran empresa de una misma cadena de valor.

- El impulso a la creación de centros de desarrollo tecnológico y a la generación de capacidades para la prestación de Servicios Tecnológicos en Parques Industriales y/o Sectores Industriales Planificados.

- La promoción de la protección de los resultados innovativos (patentes) producto de la actividad de I+D en diferentes ramas de la actividad científico-tecnológica.

- El fomento de la desregulación de productos biotecnológicos.

- La ayuda a empresas a identificar y solucionar retos tecnológicos que afecten su competitividad mediante el apoyo de asesores tecnológicos expertos en su sector de actividad (asistencias tecnológicas). 
LOS INSTRUMENTOS DE LA POLÍ́TICA EN CIENCIA,

TECNOLOGÍA E INNOVACIÓN EN LA ARGENTINA RECIENTEA

110 El estímulo a la incorporación de profesionales altamente calificados con título de doctor al sector productivo de manera permanente.

Otra forma que revisten los instrumentos para el sector empresarial son los créditos con bonificación o baja tasa; son créditos bancarios cuya tasa de interés se encuentra bonificada o reducida a través de la asignación de fondos públicos. En este caso el organismo del ámbito de la CTI se asocia con un banco comercial con el propósito de mejorar la competitividad de empresas productoras de bienes y servicios a través de la modernización tecnológica de productos o procesos. En el caso argentino se observa un crédito específico para la iniciación o consolidación de la actividad exportadora de empresas PyME de la Industria del Software.

Por último, otra forma que toman los instrumentos para este sector son los beneficios impositivos/ fiscales que incluye el otorgamiento de exenciones, deducciones de la base imponible y reducción de alícuotas basándose en tratamientos especiales que se apartan de lo establecido con carácter general en la legislación tributaria y previsional. En el caso argentino, puede mencionarse el Crédito Fiscal para Modernización Tecnológica que el FONTAR viene implementado anualmente desde el año 2002, a través del cual la empresa puede aplicarlo al impuesto a las ganancias. La mayoría de los instrumentos que poseen como destinatario a las empresas son implementados por el FONTAR; los restantes son puestos en marcha por el FONSOFT (ANR para el sector TIC) y por el MINCYT.

En el caso de los instrumentos destinados al sector académico se caracterizan por ser subsidios de proyecto o subsidio a la investigación. Este se ha convertido en un instrumento de significativa relevancia, no solo debido a los volúmenes de recursos que involucra, sino también a algunas de las características que posee. Específicamente, se define subsidio de proyecto como el dinero atribuido a un grupo o un individuo para realizar una actividad de investigación de alcance, presupuesto y tiempo limitado, normalmente Vol. 10 • Núm.18 / ene-jun / 2018 sobre la base de la presentación de una propuesta que describe las actividades de investigación por hacer (Lepori at. al., 2007). Ahora bien, los objetivos de los subsidios al sector académico son de amplia diversidad. En el caso argentino encontramos subsidios destinados a:

- La generación de nuevos conocimientos, ya sea áreas específicamente mencionadas, por ejemplo, atención de la salud y práctica clínica hospitalaria, como a nivel general. Los resultados que surjan de estos proyectos están destinados a priori al dominio público y no están sujetos a condiciones de confidencialidad comercial.

- La formación a nivel doctoral de investigadores en el formato de beca.

- La organización de reuniones periódicas nacionales e internacionales en la Argentina y de encuentros para la discusión de temas de investigación específicos (talleresworkshops).

- La incorporación de investigadores científicos y/o tecnológicos formados a unidades ejecutoras, existentes o a crearse, en áreas tecnológicas prioritarias y de alto impacto económico y/o social, y o regiones geográficas prioritarias.

- La repatriación de científicos desde el exterior.

- La generación de lazos de cooperación internacional mediante pasantías o estancias de formación en el exterior y el desarrollo de proyectos en conjunto con centros de investigación extranjeros.

- La adquisición o el mejoramiento de la infraestructura y el equipamiento, la acreditación de laboratorios.

La mayor parte de los instrumentos que tienen como destinatario al sector académico son implementados por el FONCYT y el CONICET; también el MINCYT pone en marcha algunos de estos instrumentos y cada uno de los fondos tiene una bolsa de becas específica. 
Respecto a los instrumentos que están destinados a actores del sector académico y del sector empresarial, si bien estos constituyen una minoría, su presencia en la cartera de instrumentos es novedosa y por ello representó un cambio significativo en la política.

Los destinatarios de este tipo de instrumento son personas físicas o instituciones públicas y/o privadas dedicadas a la actividad científica y tecnológica y empresas del sector productivo. En algunos casos se busca la asociación entre una institución de investigación y el actor destinatario de los conocimientos generados como es el caso de los Proyectos de Investigación Científica Orientados (PICTO). En esta modalidad se requiere presentar a un socio dispuesto a cofinanciar (50\%-50\%) el proyecto junto con el organismo que implementa el instrumento.

En otros casos, se busca promover la Asociación en Aglomerados Productivos (AP), esto es, en concentraciones territoriales de empresas -con predominio de PYME-, que presentan cierta especialización productiva similar o complementaria en un mismo sector productivo o cadena de valor (vitivinícola, apícola, textil, frutas, entre otros), y entre las cuales se desarrollan -con diversos grados de intensidad y frecuencia- vínculos de cooperación e iniciativas asociativas. Tal es el caso del PITEC (Proyecto Integrado de Aglomerados Productivos) y del FIT AP (programa de Fortalecimiento de la Innovación Tecnológica en Aglomerados Productivos).

Mediante el PITEC se apunta a promover el surgimiento de acuerdos estratégicos entre los actores de un $\mathrm{AP}$, en cuanto al desarrollo de dinámicas innovadoras que contribuyan al mejoramiento de la competitividad. Mientras que, por medio del FIT $\mathrm{AP}$, se busca aumentar la inversión y fortalecer los procesos de innovación; se exige la elaboración de un Plan de Mejora Tecnológica. En ambos casos la solicitud de financiamiento es presentada mediante una Asociación Ad-Hoc en la que pueden intervenir grupos de empresas, centros de investigación y formación superior, gobiernos provinciales y/o 111 municipales, cámaras empresariales, ONG, entre otros, ubicados todos en una determinada región.

Otra novedad dentro de los instrumentos que buscan la asociación entre actores de ambos sectores son los FITR (Fondos de Innovación Tecnológica Regionales). Estos buscan promover la generación de innovaciones y de capacidades para innovar que son críticas para el desarrollo de los sectores específicos en términos de sectores y de regiones geográficas denominados, Núcleos Socio Productivos Estratégicos (NSPE), emanados del Plan Argentina Innovadora 2020. Este instrumento debe ser presentado por un Consorcio Asociativo Público-Privado (CAPP) integrado, como mínimo, por una institución pública, conjuntamente con al menos una empresa privada nacional radicada en un espacio territorial fuera de las ciudades de mayor desarrollo del país (Buenos Aires, La Plata, Bahía Blanca, Santa Fe, Rosario y Córdoba); y un grupo dedicado a investigación, desarrollo e innovación, perteneciente a la institución pública integrante del CAPP. En cuanto a los sectores estratégicos se mencionan agroindustria; industria; energía; salud; ambiente y desarrollo sustentable; desarrollo y tecnología social.

Entre los objetivos de los restantes instrumentos destinados a ambos sectores, pueden mencionarse la generación de plataformas tecnológicas, el apoyo para crear capacidades para la prestación de servicios tecnológicos en parques industriales y/o sectores industriales planificados, el desarrollo, fortalecimiento o modernización de infraestructura, equipos y capacitación de recursos humanos, la desregulación de productos agrobiotecnológicos, entre otros.

\section{c) La edad de los instrumentos}

Si se incorpora la dimensión temporal, puede aportarse al estudio un corte histórico como aspecto adicional del análisis. Si se considera la «edad» de ciertos instrumentos, es decir, el momento en que fueron puestos en marcha, puede inferirse la 
112 complejidad del esquema de promoción vigente en el ámbito CTI, en términos generales, en base a la cantidad y a la diversidad de tipos de instrumentos y organismos de implementación. Partiendo de la consideración que con la crisis del año 2001 devino un nuevo modelo económico, que paulatinamente fue estableciéndose desde 2002/2003 también afectando el esquema de fomento de la CTI, se establecieron cinco etapas o períodos que coinciden con gestiones presidenciales: el período previo a 2003, el período del gobierno al frente del presidente Néstor Kirchner (2003-2007), el primer período (2007-2011) y el segundo (2011-2015) de la presidencia de Cristina Fernández y aquel que comienza con la gestión de Mauricio Macri en el año 2016.

La primera etapa, denominada «tradicional», abarca un período que se define por anteceder al año 2003. Antes de ese año, en Argentina se habían implementado 16 instrumentos con cinco objetivos distintos. Algunos se habían creado o, al menos, vuelto a implementar a partir de 1984, luego del retorno a la democracia; otros fueron producto de la creación del FONTAR en 1992 y del FONCYT en 1996. Previo a 2003, existían cuatro fondos para promoción de la innovación y la competitividad en las empresas y dos fondos sectoriales que, particularmente, eran consejerías tecnológicas, gestionados por el FONTAR; cuatro fondos para la promoción de la investigación científica y tecnológica, uno de ellos implementado por la Secretaría de Políticas Universitarias y los restantes por el FONCYT; un fondo para la creación y mejora de equipamiento e infraestructura gestionado también por el FONCYT, y cinco programas de formación de recursos humanos implementados por CONICET.

La segunda etapa se caracteriza por una «expansión y diversificación» de instrumentos. Entre 2003 y 2007 se crearon 17 nuevos instrumentos, sumando cuatro nuevos tipos a los cinco existentes. Por un lado, el FONCYT comenzó a implementar dos instrumentos para áreas prioritarias (áreas de vacancia y áreas estratégicas) y uno para la creación y fomento de pyme (PICT Star-up), mientras que el FONTAR dio marcha al PI-TEC -mencionado previamente- un programa para la creación de clústeres, polos tecnológicos e incubadoras de empresas. De la mano de la creación del FONSOFT aparecieron los fondos sectoriales propiamente dichos: se crearon ANR, créditos y subsidios para la industria del sector software. Por otro lado, se dio impulso a dos instrumentos para la vinculación internacional: el Programa Raíces para la repatriación de investigadores argentinos y los programas para la conformación de proyectos de investigación entre Argentina y otro país u organismo extranjero.

En la tercera etapa que va desde 2008 a 2011 se evidencia una «explosión» en el número de instrumentos. Se abrieron convocatorias para 33 nuevos instrumentos, no obstante, la novedad en este período, no fue la creación de nuevos tipos de instrumentos sino el aumento de los mismos dentro de categorías ya existentes. Es de destacar la creación de nueve fondos sectoriales, siete programas para la formación de recursos humanos y seis para la mejora del equipamiento y la infraestructura.

A partir del 2008, comenzaron a ganar terreno los instrumentos que tendieron a promocionar sectores específicos, que se destacaron dentro de una necesaria sustitución de importaciones o como nuevos segmentos estratégicos desde el punto de vista de la capacidad de innovación (Nanotecnología, Biotecnología, TIC, Energía) y de la creación de empleo (agroindustria, salud, energía, desarrollo social, medio ambiente y cambio climático). Asimismo, los nuevos instrumentos, como los programas de capacitación técnica, así como los aquellos dentro de la categoría de formación de recursos humanos, también se dedicaron a estas áreas. Se crearon las escuelas en nano y en biotecnología, las pasantías profesionales IEASTE, las becas TIC; una bolsa de becas en fondos sectoriales, un programa del FONTAR para recursos 
humanos altamente calificados y otro del FONCyT y un programa del MINCyT para la formación de recursos humanos para los Sistemas Nacionales y para la gestión de la CTI. También los instrumentos para el desarrollo de infraestructura y equipamiento estuvieron orientados a áreas estratégicas: el FONARSEC implementó los PRIETEC, el FONCyT, los PRAMIN, el MINCYT, el «Programa de mejora de equipos de los Sistemas Nacionales» y el de «Seguridad e higiene y acreditación de laboratorios».

Tabla 2. Etapas en la implementación de instrumentos de política CTI

\begin{tabular}{|c|c|c|c|c|c|}
\hline Etapa & Período & Tipos de Instrumentos & $\begin{array}{l}\text { Organismos de } \\
\text { implementación }\end{array}$ & $\begin{array}{c}\text { Cantidad } \\
\text { de nuevos } \\
\text { instrumentos }\end{array}$ & $\begin{array}{l}\text { Cantidad total } \\
\text { de instrumentos } \\
\text { en el período }\end{array}$ \\
\hline Tradicional & $\begin{array}{l}\text { Previo a } \\
2003\end{array}$ & $\begin{array}{l}\text { Implementación de instrumentos } \\
\text { tradicionales. }\end{array}$ & $\begin{array}{l}\text { CONICET, SPU } \\
\text { FONTAR, FONCYT }\end{array}$ & 16 & 16 \\
\hline $\begin{array}{l}\text { Expansión y } \\
\text { diversificación } \\
\text { de instrumentos }\end{array}$ & 2003-2007 & $\begin{array}{c}\text { Aumenta al doble el número de } \\
\text { instrumentos y aparecen nuevos } \\
\text { tipos. }\end{array}$ & $\begin{array}{l}\text { CONICET, SPU } \\
\text { FONTAR, FONCYT } \\
\text { FONSOFT }\end{array}$ & 17 & 33 \\
\hline $\begin{array}{l}\text { Explosión de } \\
\text { instrumentos }\end{array}$ & 2008-2011 & $\begin{array}{l}\text { Aumenta y se crean instrumentos } \\
\text { en sectores estratégicos. }\end{array}$ & $\begin{array}{c}\text { CONICET, SPU } \\
\text { FONTAR, FONCYT } \\
\text { FONSOFT, FONARSEC } \\
\text { MINYCT }\end{array}$ & 33 & 66 \\
\hline $\begin{array}{l}\text { Expansión de } \\
\text { instrumentos }\end{array}$ & 2012-2015 & $\begin{array}{c}\text { Aumenta y se crean instrumentos } \\
\text { para la asociación público/ } \\
\text { privada }\end{array}$ & $\begin{array}{c}\text { CONICET, SPU } \\
\text { FONTAR, FONCYT } \\
\text { FONSOFT, FONARSEC } \\
\text { MINYCT }\end{array}$ & 28 & 94 \\
\hline $\begin{array}{l}\text { Moderado } \\
\text { aumento de } \\
\text { instrumentos }\end{array}$ & $\begin{array}{l}\text { Posterior } \\
\text { a } 2016\end{array}$ & $\begin{array}{l}\text { se crean nuevos instrumentos } \\
\text { dentro de los tipos ya existentes }\end{array}$ & $\begin{array}{c}\text { CONICET, SPU } \\
\text { FONTAR, FONCYT } \\
\text { FONSOFT, FONARSEC } \\
\text { MINYCT }\end{array}$ & 3 & 97 \\
\hline
\end{tabular}

Fuente: elaboración propia.

Entre 2011 y 2015 se crearon 28 nuevos instrumentos. Solo se crearon dos nuevos tipos: los programas de apoyo a la incorporación de investigadores y becarios en empresas del MINCYT y una estructura de interfase aplicada por el FONCYT. La novedad en esta etapa fue el impulso a aquellos instrumentos que buscan la asociación ente actores públicos y privados. Desde el FONTAR creó los FIT-AP, el FONCYT el programa de subsidios a Proyectos Concertados con Empresas, el FONARSEC puso en marcha el FITR mencionado anteriormente, el MINCYT los proyectos asociativos de diseño. Asimismo, desde el MINCYT se implementaron una serie de instrumentos para la promoción de la incorporación de investigadores y becarios en empresas $y$, desde el FONTAR se abrieron nuevas líneas de crédito para la mejora de la competitividad de las empresas en el marco del Plan Argentina Innovadora 2020 y, en conjunto con bancos comerciales. La figura del consorcio o de la asociación ad-hoc fue la novedad de esta etapa.

Finalmente, se mencionan los instrumentos creados durante el primer año de gobierno del Presidente Macri. En 2016, se abrieron nuevas convocatorias para ANR para desarrollos tecnológicos con impacto social, para la regulación de productos biotecnológicos y para proyectos estratégicos en tres áreas: Alerta Temprana de Catástrofes, Desarrollos Satelitales y Cultivo Marino.

Este ejercicio de realizar la descripción cualitativa de los instrumentos introduciendo una dimensión 
LOS INSTRUMENTOS DE LA POLÍ́TICA EN CIENCIA,

TECNOLOGÍA E INNOVACIÓN EN LA ARGENTINA RECIENTEA

114 temporal, robustece el análisis de la política argentina CTI, aunque aún requiere de una mayor profundización y exploración de dimensiones no contempladas. Tal es el caso de los efectos en la creación o puesta en marcha de los instrumentos y la vinculación con el financiamiento internacional.

\section{CONCLUSIONES}

Este trabajo se propuso explorar la política en CTI reciente en Argentina, a partir de distinguir y analizar sus instrumentos. Luego de un recorrido por la literatura que ha empleado el término instrumentos de política pudo advertirse que constituye una noción ampliamente utilizada. Diversos académicos, asícomo distintos organismos internacionales y regionales han buscado generar bases de datos que recopilen y clasifiquen las diversas iniciativas de política en CTI. Sin embargo, el avance en el análisis de los instrumentos no se ha traducido en un progreso significativo del conocimiento de las políticas en CTI, poniendo de manifiesto la necesidad de desarrollar marcos analíticos y orientaciones metodológicas más amplias y más complejas que las disponibles hasta el momento.

Este trabajo ha buscado realizar un aporte analítico a la agenda de investigación de las políticas públicas en CTI, a través de la explorar los instrumentos seleccionados por los gobiernos argentinos para alcanzar determinados objetivos, pero haciendo hincapié en tres cuestiones. Respecto a los organismos encargados de la implementación de los instrumentos, se obtiene que existe una concentración en la ANCPYT, esta, a través de sus cuatro fondos, implementa una gran cantidad de instrumentos con una amplia diversidad de objetivos. Es de notar la relevancia que posee el MINCYT, organismo encargado de la formulación de la política, pero que con el transcurso del tiempo se ha convertido en agencia ejecutora de políticas. En relación con CONICET, el organismo más antiguo del complejo de instituciones de CTI en Argentina, se advierte un sesgo en sus instrumentos debido a una recolección limitada de información desde la plataforma. Algo similar sucede con la SPU, agencia que se ve incluida por la aplicación de un solo instrumento pero que en sus actividades cuenta con otras diversas iniciativas.

En relación con los destinatarios de los instrumentos, puede decirse que, si bien en la política en CTI de Argentina siguen predominando los instrumentos destinados al sector académico, aquellos que buscan afectar la situación de la CTI en el ámbito empresarial o productivo poseen una importancia relativa. Más aún, la información referida a los objetivos de cada instrumento arrojó como resultado la disponibilidad de instrumentos para las distintas etapas del proceso de mejora de la competitividad de las empresas.

La novedad en cuanto a los destinatarios resulta ser la asociación entre empresas, entre empresas y centros de investigación, y entre actores públicos y privados que se busca con ciertos instrumentos. De este modo, es la propia política que construye una población objeto del instrumento que no existe en la realidad, sino que debe ser conformada para solicitar el financiamiento. La asociación es vista, de este modo, como un elemento valioso en la generación del proceso de innovación. Este tipo de instrumentos puede representar un cambio en la gestión de la política de CTI desde un pensamiento lineal a un pensamiento sistémico sobre la I+D. Puede decirse que se da una transformación desde instrumentos centrados principalmente en actores individuales, a aquellos que intentan desarrollar vínculos nuevos o más fuertes entre los diversos actores.

Por último, se ha buscado indagar en el momento de creación de los instrumentos con el propósito de advertir tendencias en la política. En una aproximación inicial pudieron identificarse distintas etapas en la implementación de instrument os de la política de CTI. En términos generales, ha habido un aumento sostenido del número y de la diversidad de los instrumentos que se explica, en primer lugar, por un aumento de la inversión en I+D. En 
segundo lugar, dicho proceso debe entenderse en el marco de un aprendizaje institucional y de un proceso de evolución de las políticas en materia de CTI. La experiencia internacional muestra que, después de un cierto periodo de aplicación de políticas horizontales, muchos países han iniciado una transición hacia políticas más específicas, que la literatura especializada sugiere como parte de la evolución necesaria hacia mayores políticas de impacto de acuerdo con la madurez de los procesos de innovación. Se evidenció a través de estos programas que, durante este nuevo patrón económico, la política de promoción intentó corregir la falta de especificidad y complejidad en el diseño de los instrumentos.

\section{REFERENCIAS}

Albornoz, M. y Gordon, A. (2010). La política de ciencia y tecnología en Argentina desde la recuperación de la democracia (1983-2009), en Albornoz, M. y Sebastián, J. (eds.). Trayectorias de las políticas científicas y universitarias en Argentina y España, 67-122. Madrid: CSIC.

Aristimuño, F. J. y Aguiar, D. (2015). Construcción de las políticas de ciencia y tecnología en la argentina (1989-1999). Un análisis de la concepción de las políticas estatales. Redes, 21(40), 41-80.

Baptista, B. (2016). Los instrumentos de política de ciencia, tecnología e innovación en América Latina. En El estado de la ciencia. Principales Indicadores de Ciencia y Tecnología Iberoamericanos/ Interamericanos 2016. RICYT-OEI-UNESCO.

Calderari, M.; Casalet, M.; Fernández, E. y Oteiza, E. (1992). Instituciones de promoción y gobierno de las actividades de investigación. En La política de investigación científica y tecnológica argentina, (Dir.) Oteiza, E. Buenos Aires: Centro Editor de América Latina.

Centro Interdisciplinario de Estudios en Ciencia, Tecnología e Innovación (CIECTI). (2015). Lineamientos estratégicos recientes de políticas de CTI en países emergentes: reflexiones y lecciones para argentina políticas CTI en países emergentes. 115 Análisis comparado de experiencias heterogéneas y su aplicabilidad en Argentina. Recuperado de http://www.ciecti.org.ar/wp-content/ uploads /2016/09/CIECTI-Proyecto-GrupoREDES.pdf

Emiliozzi, E. (2012). Políticas en Ciencia y Tecnología y Universidad en Argentina. Análisis sobre la formación e inserción de los recursos humanos calificados. VII Jornadas de Sociología de la UNLP, Argentina en el escenario latinoamericano actual debates desde las ciencias sociales. La Plata.

Emiliozzi, S.; Lemarchand, G. A. y Gordon, A. (2009). Inventario de Instrumentos y Modelos de Políticas de Ciencia Tecnología e Innovación en América Latina y el Caribe. Working Paper 9. Banco Interamericano de Desarrollo.

Hurtado, D. (2010). La ciencia argentina: un proyecto inconcluso: 1930-2000. Buenos Aires: Edhasa.

Lemarchand, G. (2011). Sistemas nacionales de ciencia, tecnología e innovación en América Latina y el Caribe. Estudios y documentos de política científica en ALC, Vol. 1. Montevideo: UNESCO.

Lemarchand, G. (2015). Capítulo 7: Latin America. En UNESCO Science Report: Towards 2030, UNESCO: París.

Lepori, B.; Van den Besselaar, P.; Dinges, M.; Van der Meulen, B.; Potì, B.; Reale, E. y Theves, J. (2007). Indicators for comparative analysis of public project funding: concepts, implementation and evaluation. Research Evaluation, 16(4), 243-255.

Lowi, T. (1964). American Business and Public Policy Case Studies and Political Theory. World Politics, 16(4), 677-715.

Mallo, E. (2011). Políticas de ciencia y tecnología en la Argentina: la diversificación de problemas globales, ¿soluciones locales? Redes, 17(32), 133-160. 
LOS INSTRUMENTOS DE LA POLÍ́TICA EN CIENCIA,

TECNOLOGÍA E INNOVACIÓN EN LA ARGENTINA RECIENTEA

116 Porta, F. y Lugones, G. (Dirs.). (2011). Investigación científica e Innovación tecnológica en Argentina. Bernal: Editorial de la Universidad Nacional de Quilmes.

Sagasti, F. y Aráoz, A. (1976) Science and Technology Policy Implementation in Less Developed Countries: Methodological Guidelines for the STPI Project. International

Development Research Centre: Ottawa.

Sagasti, F y Araoz, D. (1979). Financiamiento del desarrollo de la ciencia y tecnología en el Tercer Mundo. Nueva Sociedad, 42(3), 15-33.

Sagasti, F. (2015). Looking Back to Move Forward: A forty-year retrospective of the Science and
Technology Policy Instruments (STPI) Project. Foro Nacional Internacional: Lima.

Sarthou, N. (2015). Los instrumentos de política como enfoque de análisis de los sistemas de pago al mérito contribuciones analíticas a partir del caso argentino. Perfiles Educativos, XXXVII(149), 150-168.

Sarthou, N. (2016). Twenty Years of Merit-Pay Programme in Argentinean Universities: Tracking Policy Change through Instrument Analysis. Higher Education Policy, 29(3), 379397.

Souza, C. (2006), Políticas públicas: uma revisão da literatura. Sociologias, 8(16), 20-45. 